\title{
OPTIMAL INVESTMENT STRATEGIES FOR RENEWABLE FACILITIES
}

\author{
JOE FLOOD \\ Australian Housing and Urban Research Institute, \\ Level 7, 20 Queen St Melbourne 3000, AUSTRALIA.
}

\begin{abstract}
Although a large literature exists on the repair and deterioration of machines, the associated problem of maintenance schedules for deteriorating renewable facilities has been little studied. These facilities include all those which can be restored to a near-new state by renovation or rebuilding, so that the market value and performance of the facility depends on the current state of repair rather than on the time since initial construction. This paper solves the general deterministic problem of finding the optimal repair strategy for a depreciating renewable facility. It is shown that the value of the facility should approach the level where a function defined as the "nett internal return" is greatest. If the facility has a finite life before sale or demolition, an adjustment to repair strategies should be made as the facility approaches this time, increasing repairs where this permits a better sale price to be obtained, or discontinuing repairs if they are not justified by scrap or market value. Solutions for a range of common depreciation functions and for linear and quadratic repair cost functions are obtained. The optimal life of the facility is determined at the time when nett "external" marginal return, which includes potential capital gain or loss and opportunity cost of capital, falls to zero.
\end{abstract}

Keywords: Replacement strategies, renewable facilities, depreciation, optimal control

\section{Introduction}

A very large literature has developed around the problem of determining optimal maintenance and replacement strategies for deteriorating facilities, as the review articles by Pierskalla and Voelker (1976) and Pintolon and Golders (1992) relate, or as detailed in texts such as Jardine (1970, 1973), Rapp (1974), Gertsbakh (1979), and Newnan (1983). A range of techniques has been developed for examining the economic lifetimes of machines in particular, ranging from the deterministic analyses of Hotelling (1925), Naslund (1966), Thompson (1968) and Hartl (1981), through statistical control (Kamien and Schwartz 1971, Virtanen 1982, Hopp and Wu 1990, Cho 1993, Tang 1993, Mehrez and Bernan 1994), to reliability theory (Barlow and Proschan 1975, Gertsbakh 1989, Chand and Sethi 1982).

The problem of deteriorating built facilities or infrastructure is rather different from deterioration of a machine, in that many facilities may be restored to near-new state by rebuilding or renovation. The value and performance of these facilities depend on their current state of repair rather than on the time since initial construction, as with a machine. Those facilities which have a large number of small components which can be regularly 
replaced without affecting system integrity, or which have a basic structure with a very long life, are most typical. However, any facilities which require maintenance, produce an income, and can be sold on the market might be considered using this type of analysis; ranging from machines and automobiles, factories, buildings, rental housing, forestry plantations and computer installations to large scale networked infrastructure such as electricity or telephone networks.

Lesse and Roy (1987) treated repair of deteriorating infrastructure as analogous to operation of a renewable resource, developing the system as an autonomous linear control problem, while making special assumptions about the form of the cost and depreciation functions. The form of solution depends a good deal, however, on assumptions about the form of the deterioration function and of the repair cost function. This paper addresses the general range of functional forms which are encountered in practice while developing some alternative solution methods for the problem.

The paper sets out to develop a general solution to the deterministic problem of the optimal maintenance schedule for a deteriorating renewable facility. Initially all parameters are taken to be time-invariant and based on the current condition of the facility, and criteria for optimal repair strategies are obtained by standard control theory methods, generalising the work of other authors. In the linear case, further insight is obtained by using an alternate solution method of integration by parts. Several common depreciation functions and repair cost functions are considered to show typical strategies. Then the parameters are considered to vary with time as well as condition, to include the effects of capital gains or obsolescence. Conditions for optimal disposal times are established and perfect markets considered. Finally some comments on the form of functions and the practical value of the results are made.

\section{Renewable facilities and optimal repair strategies}

It is presumed that the current condition of a depreciating renewable facility is determined by a single variable $x$, which determines its market capital value or scrap value $p x$, with unit price $p$, and that the facility earns nett rental income $r(x)$. The facility depreciates at a rate $D(x)$ dependent on the current condition. Investment or maintenance can be undertaken to improve or restore the condition of the asset at a rate $m(t) \geq 0$ which is to be determined at different times, with a cost $c(x, m)$ which is dependent both on the amount of maintenance and the current condition.

The framework is quite general in that it is presumed that rents, capital prices and costs of maintenance activity may have independent functional forms, being determined in different markets. The forms of the functions may be difficult to determine and subject to 
considerable statistical noise. However, some general observations may be made as to how each function should be defined in practice.

Rent The function $r(x)$ is rent or income nett of all fixed costs not related to maintenance (eg nett of administration, taxes, finance etc but not depreciation), and even nett of any routine maintenance items which do not affect the value of the property. Rates of rental return or income are often liked directly to capital value, but can in fact vary with conditions in the capital and rental markets; for example, the rate of return on residential rental property (even nett of maintenance) tends to be higher for cheaper, older dwellings, and tends to vary markedly with location.

Depreciation The depreciation rate $D(x)$ should reflect the market price for facilities of different ages, and which have been subject only to routine maintenance. The standard forms of depreciation in use in accounting are "prime value" (constant, or straight-line) and "diminishing cost" (linear), but in practice depreciation may take more complex forms, as the paper discusses. For example, automobiles depreciate rapidly in the first few years then more slowly, while large scale infrastructure tends to depreciate slowly at first, then more rapidly as reliability becomes an issue.

Maintenance cost Maintenance cost $c(x, m)$ depends both on the state of repair and on the rate of maintenance. It should include all expenditure on maintenance, replacement or improvement which affects the market value of the facility, along with any costs dependent on the rate of maintenance - including any expected loss of rental income at higher rates of maintenance, when production might have to be limited or parts of the facility might not be operational. Maintenance cost should generally be considered relative to the improvement in capital value it brings.

The cost function has partial derivatives $c_{m}>0, c_{m m} \geq 0$. This presumes diseconomies of scale in repairs, in that a higher rate of repair may result in disruptions and a loss of rental income, and may cause logistical problems in the conduct of repairs.

\subsection{The optimal repair problem}

The rate of change of condition $x$ of a renewable facility is determined by the state equation

$$
\dot{x}=-D(x)+m
$$

where $D(x)$ is the natural rate of deterioration of the asset. 
The problem is then to find the optimal maintenance schedule $m(t)$ to maximise the expected nett present value $J(x, m)$ of the facility over $T$ years, taking into account rent $r$, repair cost $c(x, m)$, and final resale value $p \cdot x(T)$ at time $T$,

$$
J=\int_{0}^{T}(r(x)-c(x, m)) e^{-b t} d t+p \cdot x(T) e^{-b T}
$$

where $b$ is the discount rate. This is subject to the state equation (1), and the constraints

$$
m \geq 0, x \geq 0
$$

In some problems there may also be other constraints, $x \leq x_{0}$ for example, so that $x$ may not exceed its new value or condition.

The usual method of solution of this type of control problem is, using the Pontryagin Maximum Principle, to form the Lagrangian of the system and take partial derivatives with respect to the control and state variables, as Pitchford and Turnovsky (1977) and many other texts detail. The Lagrangian of this system is

$$
L=\left((r-c(x, m))+\mu(-D+m)+\lambda_{1}(m-0)+\lambda_{2}(x-0)\right) e^{-b t}
$$

where $\mu, \lambda_{I}, \lambda_{2}$ are costate variables (shadow prices at current values) ${ }^{1}$.

Conditions for an optimal strategy are $\dot{\mu}=b \mu-\frac{\partial L}{\partial x}, \frac{\partial L}{\partial m}=0$

(see, for example, Pitchford and Turnovsky 1977, Chapters 1 and 2).

so that,

$$
\dot{\mu}=\left(b+D^{\prime}\right) \mu-r^{\prime}+c_{x}-\lambda_{2}
$$

and

$$
-c_{m}+\mu+\lambda_{1}=0
$$

with $\lambda_{1}, \lambda_{2}$ non-zero only when the respective constraints are operational. The shadow price variable $\mu$ is the marginal benefit of conducting extra repairs, consisting of both

\footnotetext{
${ }^{1}$ These adjusted costate variables are obtained by multiplying the "true" or discounted costate variables by $e^{b t}$
} 
improved rents and extra capital value, and it usually determines both the level of repairs and whether repairs should be undertaken at all, with repairs not undertaken when $\mu<c_{m}$.

Internal stationary solutions $\left(\dot{x}=0, \dot{\mu}=0, \lambda_{1}, \lambda_{2}=0\right.$ ) occur when $\mu=c_{m}, m=D(x)$, so that (using Equations (1), (5) and (6))

$$
\left(r-\left(c(x, D(x))^{\prime}-b c_{m}(x, D(x))=0\right.\right.
$$

This equation may be solved to find $x=x^{*}$ at which the optimum applies.

Whether $x^{*}$ will provide a maximum or a minimum return depends on the second variation of Equation (2). The Legendre condition (Pierre 1969, p.115) states that $x *$ will be a maximum if $g_{x x}-d / d t g_{x x} \geq 0$ everywhere, where

$$
\begin{gathered}
g(x, \dot{x}, t)=(r+c(x, \dot{x}+D)) e^{-b t} \text { is the integrand in Equation (2). This reduces to } \\
\left(r^{\prime}-c(x, D(x))^{\prime}-b c_{m}(x, D(x))\right)^{\prime}<=0
\end{gathered}
$$

for all $x$, when $x$ is stationary.

Equations (7) and (8) may be combined into a single criterion. Maximal returns are possible at maxima of the function

$$
I(x)=r(x)-c(x, D(x))-b C(x)
$$

if this function is concave to the origin,

where $C(x)=\int_{x_{1}}^{x} c_{m}(\underline{x}, D(\underline{x})) d \underline{x}$ is the amount of money required to restore the facility

to value $x$ from some base state $x_{1}$ (eg $x_{1}=0$, or $x=x_{0}$, the initial value ${ }^{2}$, with repairs conducted exactly at the margin to arrest depreciation ${ }^{3}$.

The function $I(x)$ can be taken as the "nett internal" or recurrent rate of return. If $x$ is to be maintained at some constant value, the terms of $I$ include rent, cost of arresting depreciation, and opportunity cost of capital expenditure on repairs.

\footnotetext{
${ }^{2}$ It is sometimes convenient to presume $x_{l}=0$, for convenience in calculating $I$, as is done in this paper, and sometimes to presume $x_{1}=x_{0}$, so that $C\left(x_{0}\right)=0$ in Equation (10). The results will only differ by a constant.

${ }^{3}$ Although this function is important in the subsequent analysis, representing the cost of rebuilding a facility using on-site maintenance as opposed to its market value, it does not seem to have appeared previously in the literature of linear control.
} 


\subsection{The linear maintenance cost case and integration by parts}

Often in practice a linear form for the cost of maintenance is reasonable in the vicinity of the optimum. All other previous authors including Naslund (1966), Thompson(1968), Lesse and Roy (1987) and Tang (1993) have presumed a cost function linear in $m$, $c(x, m)=c(x) m$, with $c$ usually constant. The marginal repair cost function $c(x)$ in this case directly represents the extra value to the property through repairs, or the "repair efficiency"; for example, if $c(x)=2 p$, then half the cost of repairs at $x$ can be recouped in improved capital value.

In this linear case a bang-bang control results, and for a solution to exist, there must be an upper limit to the rate of maintenance as well as a lower limit. This maximum maintenance rate $m_{\max }$ can be taken to be the point where rents start to be seriously affected by maintenance and second-order effects or diseconomies of scale come into play ${ }^{4}$. Then maintenance either varies between minimal effort (or zero) when $x$ is too high for maximal returns, and maximal effort when it is too low.

The significance of $I(x)$ becomes more clear in the linear case when an alternative solution is obtained using integration by parts. Then,

$$
\begin{aligned}
J(x, \dot{x}) & \left.=\int_{0}^{T} r(x)-c(x)(\dot{x}+D)\right) e^{-b t} d t+p x_{T} e^{-b T} \\
& =\int_{0}^{T}(r-c D-b C) e^{-b t} d t+\left(p x_{T}-C\left(x_{T}\right)+C\left(x_{0}\right)\right) e^{-b T}
\end{aligned}
$$

The integrand in the first term is $I(x)$. Over an infinite period (or if $c(x)=p$ for all $x$, so that repair costs can always be recouped on sale), the second term will vanish. Then the problem reduces to maximising $\int I(x) e^{-b t} d t$, which will be achieved by moving rapidly towards a maximum of $I(x)$.

\subsection{Finite time horizons and transversality}

For an infinite-horizon problem ( $T$ very large) the problem can be solved by allowing the condition of the facility to move towards a maximum of $I$. A finite horizon problem is

\footnotetext{
${ }^{4}$ Alternatively, a budget constraint may be placed on maintenance $\operatorname{cost} c(x) m$, which leads to a similar solution.
} 
more complex, usually requiring some adjustment of the condition of the facility towards the end of its life to facilitate resale.

As well as Equations (5) and (6), the variable $\mu$ must satisfy a transversality equation or final condition $\mu(T)=p$. This final condition tends to determine the size of $\mu(t)$ for all $t$, determining in turn the level of repairs. The problem then is to find an initial value and a path for $\mu$ determined by the state equation (5) leading to final $\mu=p$. Repairs are conducted, according to Equation (6), whenever marginal benefits $\mu$ of repair exceed marginal costs $c_{m}$.

From Equation (10) it can be seen ${ }^{5}$ that if $I\left(x^{*}\right)$ is at a maximum, but $\mu^{*}=c\left(x^{*}\right)$ is not equal to $p$, then a final adjustment in $x$ should take place in order to reap the full benefits of sale. If $\mu^{*}<p$, then repairs can be more than recouped on sale ("cosmetic" repairs) and the facility should be improved. If $\mu^{*}>p$, the facility is underpriced, and repairs should be discontinued prior to sale.

A typical solution, then, is to move the facility towards a point of maximum return as expressed by the function $I(x)$, near where it is sustained for a time, and then improve the facility to improve or allow it to run down further prior to sale, depending on market conditions. However, this final adjustment may occupy much or all of the life of the facility, as some of the following examples will show.

\section{Some examples}

Some examples are now considered which show the utility of employing the internal return function $I(x)$ to determine solutions of the problem for different forms of the depreciation function or of the rent or repair cost functions. Example (1) is essentially the machine problem of Thompson (1968) and others, while Example (4) is the problem of Lesse and Roy (1979). In these examples, the internal return function provides an immediate solution for the infinite time horizon case, and provides a more general solution than those of earlier authors. Examples (2) and (3) are new problems; Example (2) introduces a quadratic repair function, to better simulate the diseconomies of scale in conducting repairs at a faster rate. The control solution is then shown to be a continuous "smoothed" approximation to the usual bang-bang linear repair strategy. Example 3 considers a convex quadratic depreciation function, representing facilities that depreciate more rapidly in price when new. In this case an internal solution is generally valid, and once again the "bang-bang" repair strategy can be smoothed by adopting a quadratic repair cost function.

\footnotetext{
${ }^{5}$ in the linear control case, but the result holds more generally when $\mu^{*}=c_{m}\left(x^{*}, D\left(x^{*}\right)\right) \neq p$.
} 


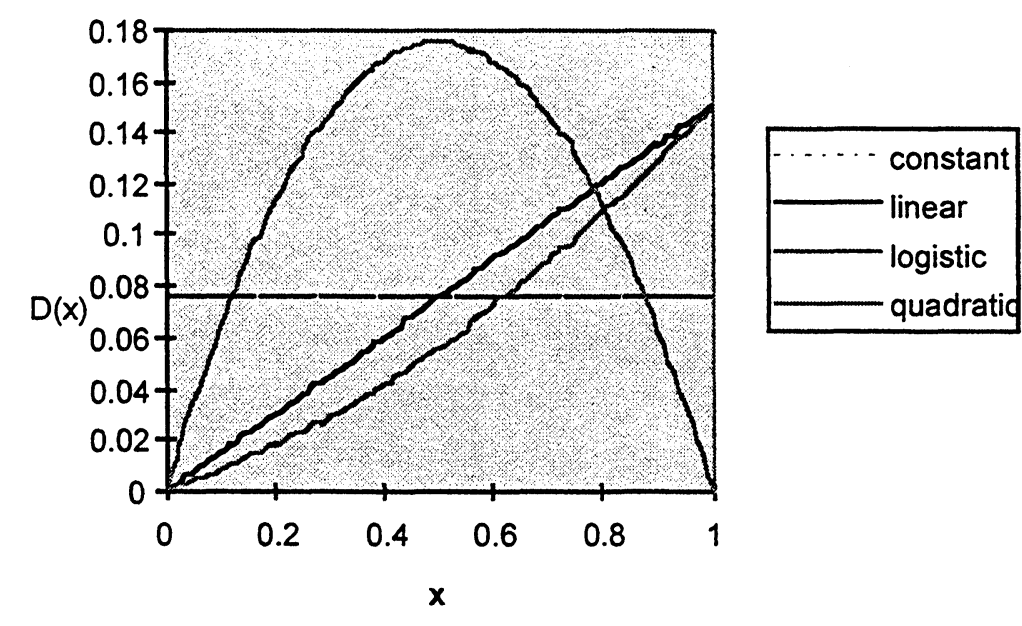

Figure 1. Depreciation functions.

The different forms of depreciation functions used in the examples are shown in Figure 1.

\section{Example 1. Linear costs, straight line depreciation.}

If depreciation is constant $D(x)=d$, and all other functions are linear, so that $r(x)=r p x$, $c(x, m)=c p m$, for constants $r, c$, this is the (time-invariant) classic machine problem of Naslund and Thompson. In this case, $I(x)=p x(r-c b)-c d$, which takes a maximum only at maximum or minimum values of $x$. Therefore, when time horizons are long, the facility should be improved or maintained in original condition if $r>c b$, that is, if rent is sufficiently high to cover the opportunity cost of capital expenditure on repairs; and allowed to depreciate otherwise. Towards the end of the period, an adjustment upwards or downwards to reflect sale values is required, depending whether $c<1$ or $c>1$.

In this particular case, the time when the strategy is to be adjusted can be easily determined. In Thompson's (1968) original solution, Equation (5) becomes

$$
\dot{\mu}=b \mu-r
$$

This simple linear d.e. is solved to give $\mu=\mu^{*}+\left(p-\mu^{*}\right) e^{-b(T-t)}$, where the steady state value $\mu^{*}=r / b$. Then $\mu$ is strictly increasing or decreasing with time according as 
whether $r$ is greater than or less than $b p^{6}$. The repair strategy will reverse at the single point of time $t$ where $\mu=c$. This time is calculated from $(T-t)=-1 / b \ln \left(\left(c-\mu^{*}\right) /\left(p-\mu^{*}\right)\right)$.

Figure 2a shows the optimum solution for $x$ and $\mu$ over a 20 year period, when $r=0.18$, $d=0.06, b=0.1, c=1.6, x_{0}=1, p=1, c_{\max }=0.15$. In curve (1), the facility rises in value while $\mu>c$ for 6 years, after which it is allowed to decline because repairs are not justified by the sale value. In the case (2) that $x$ is not allowed to rise above its initial condition $x_{0}$, then the turning point remains at the same time, but for the first 6 years, $\mu=c$ and $x$ is preserved in its initial condition.

In Figure $2 b$, where $r=0.06$ and $c=0.8$, with other parameters unchanged, the rental return is insufficient to justify repairs; however after 14 years the prospect of sale causes "cosmetic" repairs to be undertaken, lifting the sale value of the facility.

\section{Example 2. Quadratic costs, linear depreciation}

If $c$ is quadratic in $m$, so that $c(x, m)=c_{1} m+c_{2} m^{2}$, for constants $c_{1,} c_{2,}$ and $r(x)=r p x, D(x)$ $=d x$ (linear or "diminishing balance" depreciation), then $c_{m}=c_{1}+2 c_{2} m$ and $\mu^{*}=r /(b+d)$. In this case, internal solutions $x^{*}$ may exist. The stationary value $x^{*}$ can be calculated from the maximum of $I(x)=r x-(b+d) c_{1} x-(b+d) c_{2} d x^{2}$, or by noting $c_{m}\left(d x^{*}\right)=\mu^{*}$, so $x^{*}=\left(\mu^{*}\right.$ $\left.c_{1}\right) / 2 c_{2} d$. It follows that the maximum value $x^{*}$ will lie between 0 and $x_{0}$ whenever $c_{m}(0) \leq$ $\mu^{*} \leq c_{m}\left(d x_{0}\right)$. Otherwise, if $\mu^{*}<c_{1}$ the facility should be allowed to decay; or if $c_{1}+2 c_{2} d x_{0} \leq \mu^{*}$, the best returns are obtainable by improving the facility above its initial value (or preserving it in new condition if this is not possible).

Since $\mu=c_{m}$ for an internal solution, maintenance $m=\left(\mu-c_{1}\right) / 2 c_{2}$. Also,

$$
\mu=(b+d) \mu-r
$$

so $\mu=\mu^{*}+\left(p-\mu^{*}\right) e^{-(d+b K T-t)}$, similarly as the previous example, and solving for $x$ in Equation (1),

$$
x=k e^{-d t}+k_{1} e^{-(d+b)(T-t)}+x^{*}
$$

for constants $k_{1}=\frac{\left(p-\mu^{*}\right)}{2 c_{2}(2 d+b)}, k=x_{0}-x^{*}-k_{1} e^{-(d+b) r}$.

\footnotetext{
${ }^{6}$ Thompson considered only the profitable facility case $r>b p$, and presumed that maximum repairs could not fully arrest depreciation.
} 
$r=0.18, c=1.6$

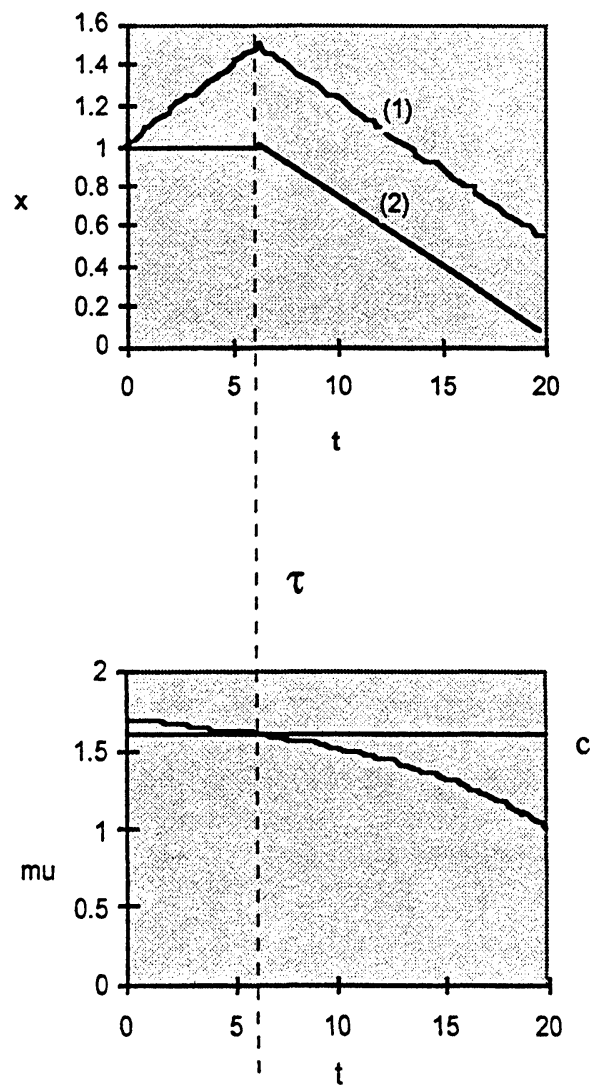

Figure 2a. Profitable facility. $r=0.06, c=0.8$

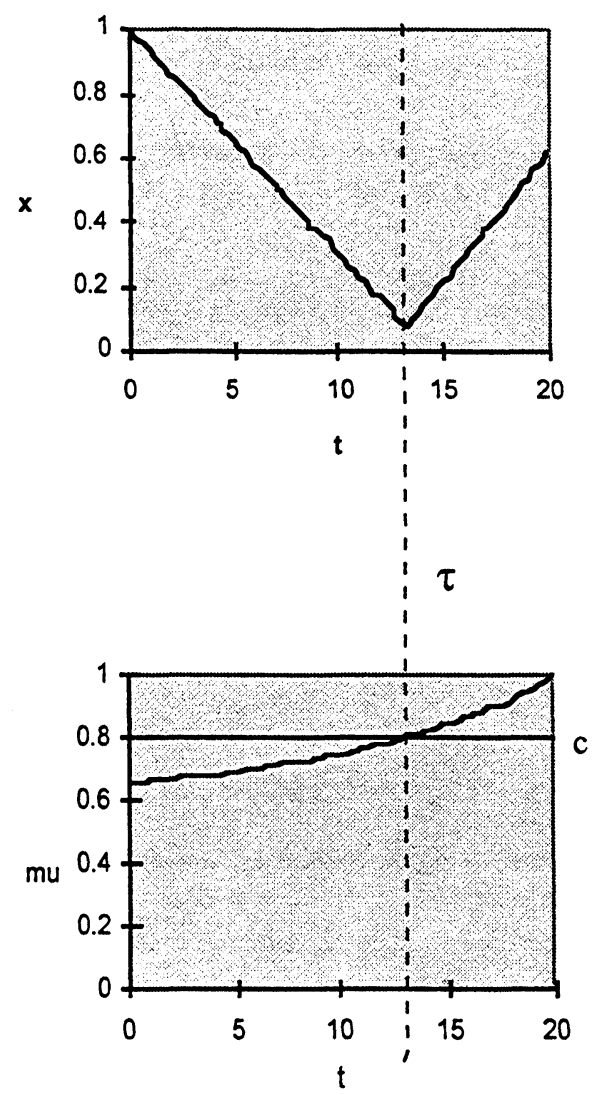

Figure 2b. Unprofitable facility, cosmetic repair.

So $x$ decays towards $x^{*}$, then adjusts towards $\mu=p$ at time $T$. If $T$ is large or $\mu^{*}=p$, maintenance should be conducted at the constant level $m^{*}=\left(\mu^{*}-c_{1}\right) / 2 c_{2}$ which arrests depreciation when $x=x^{*}$. ' Otherwise both $\mu$ and $m$ rise or fall consistently during the time period, according as $p>\mu^{*}$ or $p<\mu^{*}$.

\footnotetext{
${ }^{7}$ An alternative and possibly more easily managed strategy to the optimal strategy of conducting repairs at the constant rate $m^{*}$ is similar to that of Example 1, so that no maintenance is undertaken until the facility falls to the value $x^{*}$, where it is maintained with maintenance $m^{*}$. There is not a great difference in long-term return between the two
} 
$r=0.2, d=0.25, c 1=c 2=0.5$

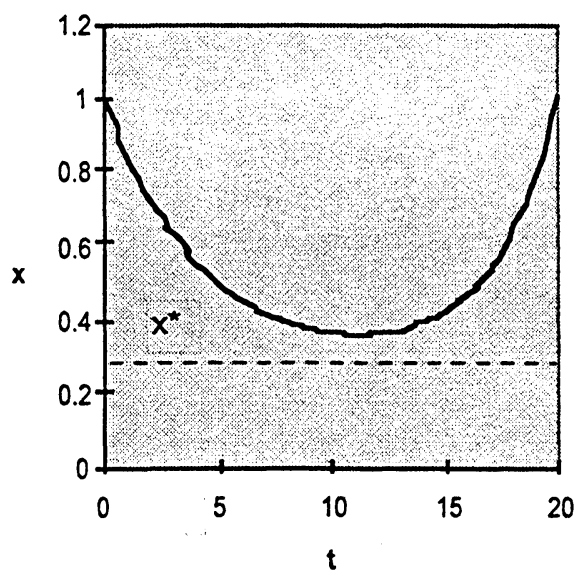

Figure 3a. Unprofitable facility, cosmetic repairs $r=0.4, c 1=0.9$

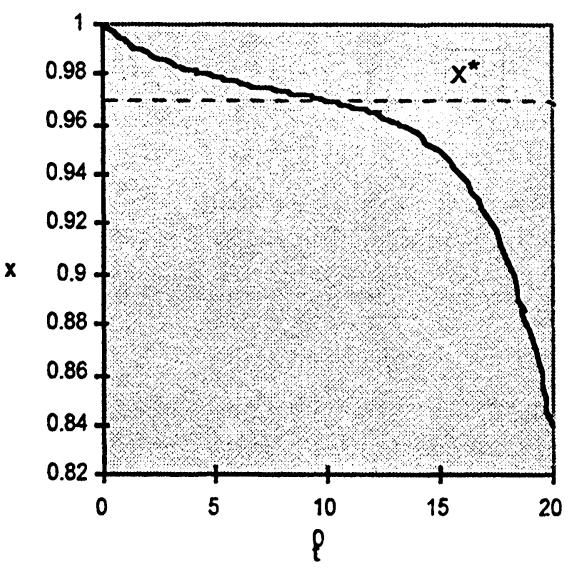

Figure 3b. Profitable facility

Figure 3a shows an "unprofitable" facility where cosmetic repairs are possible, with $r=0.2, d=0.25, b=0.1, c_{1}=c_{2}=0.5, x_{0}=1, p=1, \mathrm{~b}=0.1$. Then $\mu^{*}=0.57, x^{*}=0.29$. The repair rate rises during the life of the facility from virtually zero initially to $15 \%$ at Year 20 , bringing the facility back to its initial condition for sale. However, if $r=0.4, c_{l}=0.9$ as in Figure $3 b$, then $\mu^{*}=1.14, x^{*}=0.97$, and repair levels fall from 0.24 initially, almost arresting depreciation, to 0.11 at Year 20 .

\section{Example 4. Quadratic, concave depreciation}

The special case considered by Lesse and Roy (1987) is of linear earnings $r(x)=r x$, unit price and repair costs $p=c=1$, and quadratic depreciation $D(x)=d x\left(1-x / x_{0}\right)$, for constant $\mathrm{h}$ and initial (new) value $x_{0}$. Lesse and Roy found that in this case no internal best strategy existed. In this case, the facility should either be maintained in its initial condition, or allowed to deteriorate without maintenance, depending on the relative sizes of rates of return, repair costs, and economic discount rates.

strategies, and in fact it may take a very long time to recoup the costs of early repairs through improved rentals if the optimal strategy is used in preference. 
These results can be shown rapidly using the analysis of Section 2 . Equation (9) becomes $I(x)=(r-b) x-d x\left(I-x / x_{0}\right)$, with a minimum at $x^{*}=(d-b-r) x_{0} / 2 d$. Maxima occur only at the endpoints $x^{*}=x_{0}$ and $x^{*}=0$, so that as in Example 1, the facility should either be maintained in new condition or allowed to depreciate with minimal repair. As in that example, which of the two extreme solutions should be preferred depends on the internal returns $I\left(x_{0}\right)=(r-b)$ and $I(0)=0$. If the rent is better than the discount rate, then the facility should definitely be maintained in the best possible condition. If the rent is less than the discount rate, it would be expected that the facility should be allowed to deteriorate to $x=0$. However, if the rent is only slightly lower than the return on an equivalent investment, it may be better to maintain the facility in new condition rather than to take even lower returns while the facility depreciates.

This is typical of the situation where $I(x)$ has several local or extreme maxima. It may be preferable to move to some suboptimal maximum rather than incur extra losses of proceeding to a global maximum.

Using a quadratic cost function $c(x, m)=c_{1} m+c_{2} m^{2}$ does not alter the solution greatly, although now if $d$ is small it is possible for there to be a small local maximum of $I$ near 0 , and if $c_{2}$ is large, this may be the global maximum, as Figure 4 shows. However, approaching this maximum involves substantial losses, and it is better to keep the facility in new condition.

$d=0.1, c 10.8, c 2=22, r=0.3$

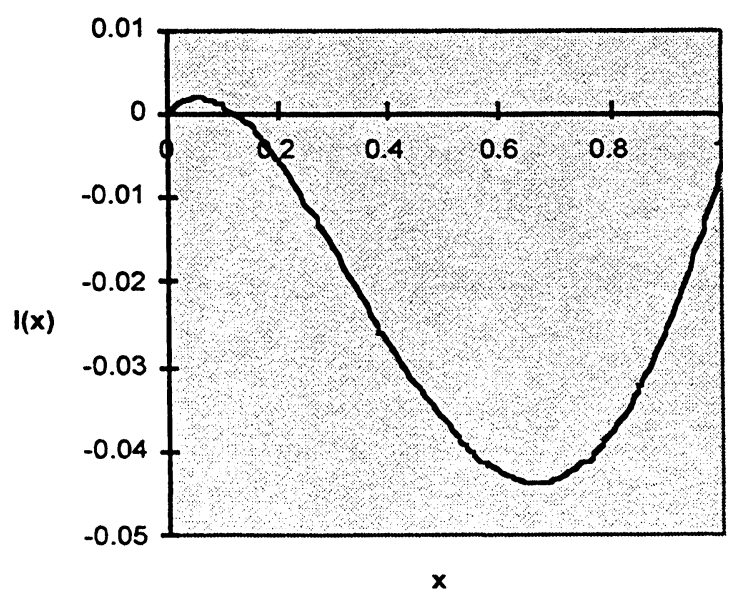

Figure 4. Internal return at different values of $x$, concave depreciation. 
While the logistic function may be a reasonable representation of reliability over time for a depreciating facility, it is not very useful in the present context. This function implies there is no cost in keeping a facility in new condition (and so this tends to be the optimum for even quite modest rent levels), when in fact maintaining perfect condition would normally be expected to require considerable outlays.

\section{Example 4. Quadratic, convex depreciation}

In contrast to the previous example, the value of capital items might be expected to depreciate most rapidly initially, so that $D^{\prime}<0, D^{n}<0$, and for these convex functions, internal points of maximal return may be found as in Example 2, even when costs are linear.

In the linear cost case, taking $D=d_{1} x+d_{2} x^{2}$, for constants $d_{1}, d_{2}$, and other functions as in Example 1, $I(x)$ has a maximum at $x^{*}=\left(r-c\left(b+d_{1}\right)\right) / 2 c d_{2}$. As Figure 5 shows, the facility should be allowed to deteriorate to this point, sustained there, and then allowed to improve or deteriorate prior to sale depending on whether $c<p$ or $c>p$.

$r=0.33, d 1=0.07, d 2=0.05, c=1.4$

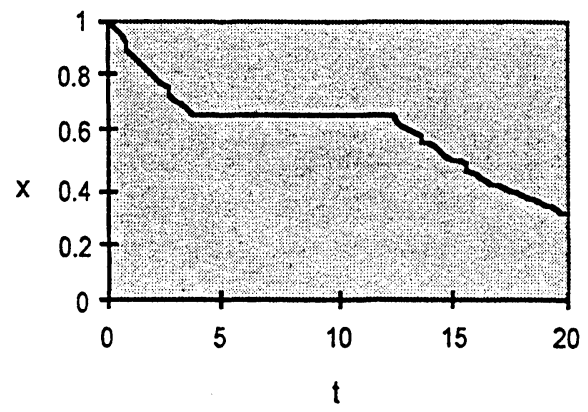

$r=0.18, c=0.8$

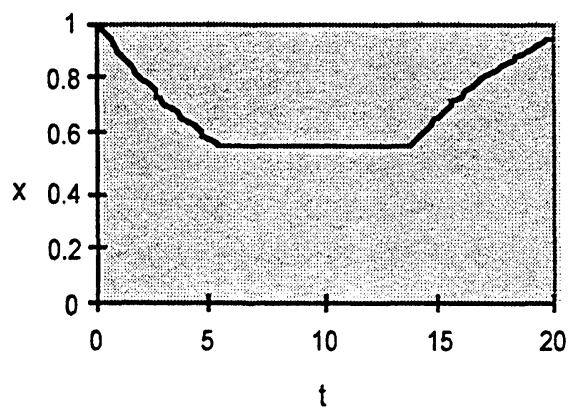

Figure 5. Facility with linear repair costs and convex depreciation.

Although this strategy is easy to apply, in fact the "bang-bang" solution is problematic at the stationary point, since either maximum or zero repairs are required when $x$ drifts from $x^{*}$. In practice, repairs are actually arrested at $x^{*}$ with $m^{*}=D\left(x^{*}\right)$.

This problem can be partially resolved using a quadratic cost function as in Example 2. Then as before, there is a single equilibrating $\left(x^{*}, \mu^{*}\right)$ where $I\left(x^{*}\right)$ is maximum (or which can also be found by solving $c_{m}\left(D\left(x^{*}\right)\right)=\mu^{*}$ and $\left(b+D^{\prime}\left(x^{*}\right)\right) \mu^{*}=r$ iteratively $)$. 

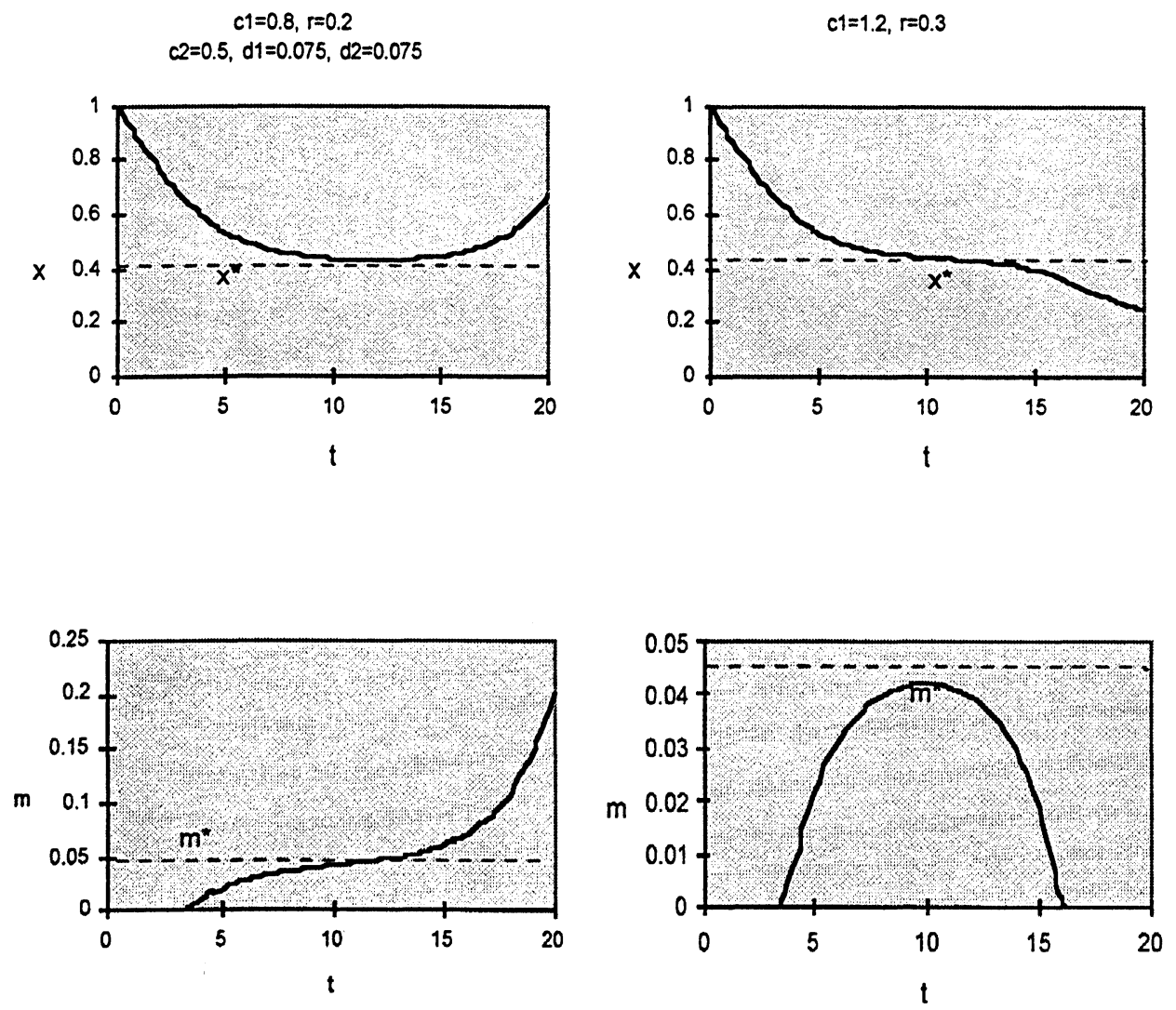

Figure 6. Quadratic cost and depreciation: $x$ and $m$ for different parameter values.

As Figure 6 shows, the results are similar to the linear case. In the first example, with low repair costs, repairs are initially nil, then are close to $m^{*}$ in the middle of the period when $x$ is close to $x^{*}$, and finally are accelerated prior to sale. In the second case with high repair costs, repairs are undertaken only in the middle of the period.

\section{Time-dependent functions and obsolescence}

There are several circumstances in dealing with a renewable facility when functions may be time-based as well as functions of the value or condition of the utility. The first occurs when the underlying structure may be subject to slow deterioration that results in a 
gradual rundown of the facility no matter what repairs are undertaken. The second concerns obsolescence.

Often a prime consideration in economic return problems is not so much the technical condition of the facility as the existence of other facilities using improved technology. If there is competition, this will lower both the return and the market value of the facility over time.

This can be accommodated in the present framework by assuming that depreciation, rental return and repair cost may be functions of time as well as condition ${ }^{8}$.

\subsection{Time-dependent control}

In this case, Equations (1) to (6) still hold. In the linear control case, an explicit solution can be obtained as before. Integration by parts yields

$$
\mathbf{J}=\int_{0}^{T}\left(r-c D-b C+C_{t}\right) e^{-b t} d t+\left(p x_{T}-C\left(x_{T}, T\right)+C\left(x_{0}, 0\right)\right) e^{-b T}
$$

where as before $C(x, t)=\int_{x_{1}}^{x} c_{m}(x, D(x), t) d \underline{x}$

The nett internal return is then

$$
I(x, t)=r-c D-b C+C_{t}
$$

where the last term allows for possible savings through avoiding future increases in costs.

The linear control solution is, again, to move towards a maximum of $I$, which will occur when $I_{x}=0$ or

$$
r_{x}-(c D)_{x}-b c+c_{t}=0
$$

If the LHS is not independent of $x$, this provides possible optimal paths for $x$ over time, above and below which minimal and maximal effort are to be applied. This is shown in Figure 7, with a typical time-dependent optimal path and adjustments prior to sale.

\footnotetext{
${ }^{8}$ Note that Naslund (1966) and Thompson (1968) are special cases where functions are only dependent on $t$ and not $x$.
} 


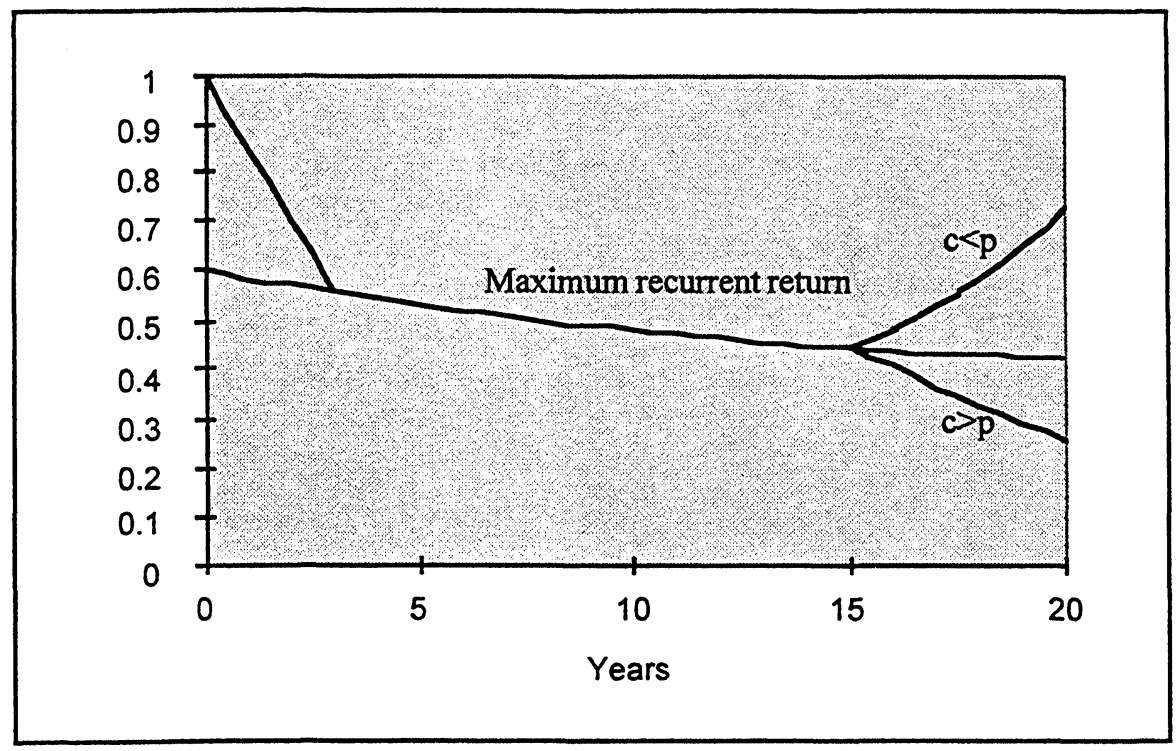

Figure 7. Repair strategy with obsolescence.

The slope of the optimal path will have the same sign as $I_{x t}$, which is the change in marginal nett return with time. It will be negative when rents are falling over time, and when repair costs are rising no faster than the discount rate.

The LHS of Equation (13) will be independent of $x$ only in the special case considered by Thompson (1968), where $r$ and $D$ are linear in $x$, and $c$ is a function of $t$ only (it will also be independent in a perfect market, as Section 4.3 shows). The control is then independent of initial values of $x$, and is applied at times determined by solving the differential equation (5) for $\mu$.

\subsection{Optimal time horizon}

Under many conditions an optimal time for retaining the facility can be determined. This can be done using another transversality condition, or by observing that the original problem can be transformed as

$$
J(x, m, T)=\int_{0}^{T}(r-c+(\dot{p} x)-b p x) e^{-b t} d t+p . x_{0}
$$


eliminating the unknown final value of $x$. Clearly, if the integrand becomes and remains negative as $T$ increases, the integral will decrease, so that a necessary condition for an optimal $T$ will be

$$
E(x, m, T)=r\left(x_{T}\right)-c\left(x_{T}, m_{T}\right)+\left(\dot{p}_{T} x_{T}\right)-b p_{T} x_{T}=0
$$

This function $E$ is what is usually known as the nett marginal rate of return. It includes rent plus capital gains less repair costs and opportunity cost of capital. Equation (15) is then a restatement of the standard result that the facility is held until such time as nett marginal return on capital, including capital gain or loss, is equal to the discount rate.

\subsection{Perfect capital market}

One particularly important example of a depreciation function occurs under perfect market assumptions. In a perfect market with perfect foresight, the marginal return on any investment is the discount rate, so that, for all $t$,

$$
(\dot{p} x)+r(x)-c(x, m)-b p x=E(x, m, t)=0
$$

calculated under an optimal repair strategy $m$. Price in the perfect market adjusts to prevent any advantage being gained at different times, and to keep returns equal to the discount rate. The nett marginal rate of return is zero at all times, and no time for sale is preferred over any other.

If $p(T) x(T)$ is known scrap value at some future time $T$, the market value $p(t) x(t)$ at any time $t$ is then

$$
p(t) x(t)=e^{b t} \int_{t}^{T}(r(x, \tau)-c(x, m, \tau)) e^{-b \tau} d \tau+e^{b(T-t)} p(T) x(T)
$$

or the present value of its nett future earnings after $t$, (as in Hotelling's (1925) original paper). This equation may be used in determining a 'nominal' market price or market depreciation for an 'unpriced' facility such as a power generating plant, where no capital market exists but nett income may be estimated.

Alternatively, rather than the capital price adjusting to maintain return, the rental market may adjust to reflect marginal costs. In this case, rentals will be set according to Equation (16) to cover marginal costs, including opportunity costs of capital, nett of capital gains. Once again, any sale time will be optimal. 


\section{Conclusions and applications}

The general problem of determining the optimal repair strategy for a depreciating renewable facility has been solved, generalising the results of other authors who have looked only at time-dependent systems or at particular examples of depreciation functions. A better insight into the qualitative nature of the solution has been obtained by using several different solution methods, including optimal control and integration by parts.

It has been shown that the facility should move towards a point or path where the "nett internal return" is greatest, and should then be repaired or allowed to decline prior to sale, depending on whether the cost of repairs can be recouped in improved capital value. Typical solutions for several cost and depreciation functions have been obtained. The time when the facility should be sold is the point where "external" marginal return (which includes capital gain) is equal to the discount rate. In a perfect capital or rental market, this will hold everywhere.

The general strategies developed in this paper can be applied to any facility as long as the appropriate functions are known. However, applying the results in practice implies a deterministic knowledge of depreciation, costs, rents and prices which is unlikely to be the case in practice. The discount rate is a partial proxy for uncertainty, but this single parameter may be inadequate to allow for changes in future conditions in determining optimal strategies. Strategies are quite sensitive to the values and form of these parameters and in practice a regular review of optimal strategies would be necessary.

A statistical approach to optimal maintenance would be a natural extension of this work. A number of authors have generalised the deterministic work on machine maintenance by Naslund (1966) and Thompson (1968), to take into account the random nature of breakdowns or of future prices, and this could also be done in the present case. However, as has occurred with stochastic approaches to machine maintenance, a knowledge of the deterministic solution is the first step in examining stochastic strategies. Also, the deterministic solution provides the necessary framework for determining the comparative static effects of government measures such as taxation and depreciation allowances upon maintenance and retirement strategies, and the companion paper Flood (1997) shows that these can be very significant.

\section{References}

1. Barlow, R. and Proschan, F. Statistical Theory of Reliability and Life Testing: Probability Models. New York: Holt, Rinehart and Winston. 1975. 
2. Chand, S. and Sethi, S.P. Planning horizon procedures for machine replacement models with several possible alternatives. Naval Research Logistics Quarterly 29, 483-493, 1982.

3. Cho, D. Optimal production and maintenance decisions when a system experiences timedependent deterioration. Optimal Control 14, pp 153-167, 1993.

4. Flood, J. Optimal replacement and taxation. To appear.

5. Gertsbakh, I.B. Models of Preventive Maintenance. Amsterdam, North-Holland, 1979.

6. Gertsbakh, I.B. Statistical Reliability Theory. New York: M Decker, 1989.

7. Hartl, R. A mixed linear-non-linear optimization model of production and maintenance for a machine, in Feichtinger G. (ed). Optimal Control Theory and Economic Analysis. Amsterdam: New Holland, 1981.

8. Hopp, W.J. and Wu, S.C. Machine maintenance with multiple maintenance choices. IIE Trans 22, pp 226-233, 1990.

9. Hotelling, H. A general mathematical theory of depreciation. Journal of the American Statistical Association (20), pp 340-353, 1925.

10. Jardine, A.K.S. Operational Research in Maintenance. Manchester: Manchester University Press, 1970.

11. Jardine, A.K.S. Maintenance, Replacement and Reliability. London: Pitman, 1973.

12. Kamien, M.I. and Schwartz, N.L. Optimal maintenance and sale age for as machine subject to failure. Management Science (17), pp B495-B504. 1971.

13. Lesse, P.F. and Roy, J.R. Optimal replacement and maintenance of urban infrastructure. Environment and Planning A (19), pp 1115-1121, 1987.

14. Mehrez, A. and Bernan, W. Maintenance optimal control: three-machine replacement model under technological breakthrough expectations. Journal of Optimization Theory and Applications (81), pp 591-618, 1994.

15. Naslund, B. Simultaneous determination of optimal repair policy and service life. Swedish Journal of Economics (68), pp 63-73, 1966.

16. Newnan, D.G. Engineering Economic Analysis. San Jose: Engineering Press Inc, 1983.

17. Pierre, D.A. Optimization Theory with Applications. New York: Wiley, 1969.

18. Pierskalla, W.P. and Voelker, J.A. A survey of maintenance models: the control of deteriorating systems. Naval Research Logistics Quarterly (23), pp 353-388, 1976.

19. Pintolon, L.M. and Golders, L.F. Maintenance management decision making. European Journal of Operational Research (58), pp 301-317, 1992.

20. Pitchford, J. and Turnovsky, S.J. (eds.). Applications of Control Theory to Economic Analysis. Amsterdam: North Holland, 1977.

21. Rapp, B. Models for Optimal Investment and Maintenance Decisions. New York: Wiley, 1974.

22. Tang, M.G. A stochastic machine maintenance and sale problem - results with different production functions. Naval Research Logistics (40), pp 677-696, 1993.

23. Thompson, G.L. Optimal maintenance policy and sale date of a machine. Management Science (14), pp 543-550, 1968.

24. Virtanen, I. Optimal maintenance policy and planned sale date for a machine subject to deterioration and random failure. European Journal of Operational Research (9), pp 33-40, 1982. 


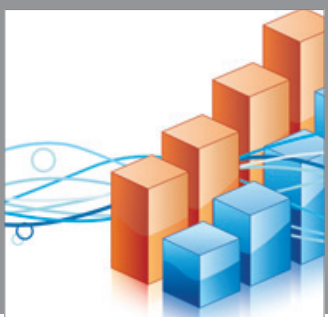

Advances in

Operations Research

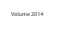

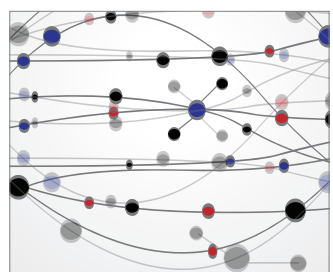

\section{The Scientific} World Journal
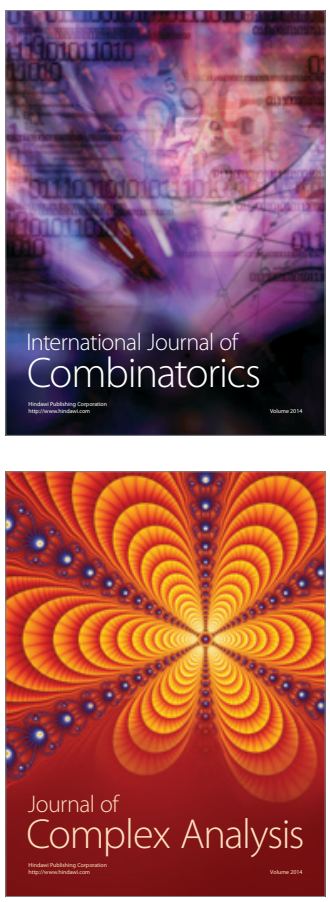

International Journal of

Mathematics and

Mathematical

Sciences
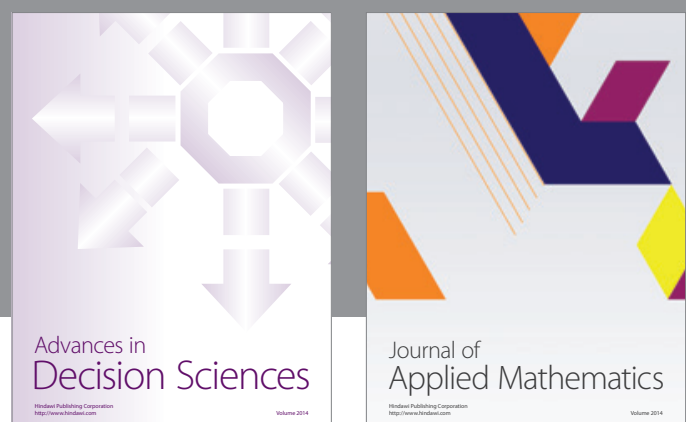

Journal of

Applied Mathematics
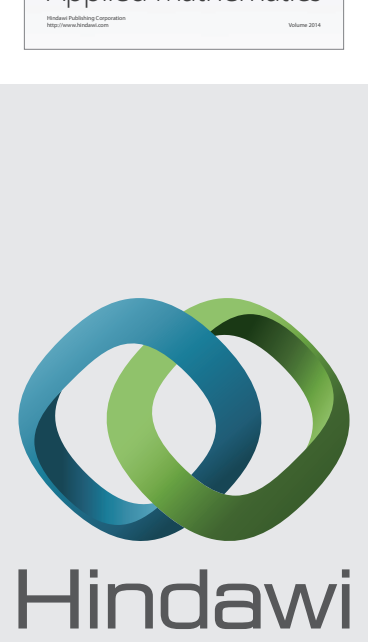

Submit your manuscripts at http://www.hindawi.com
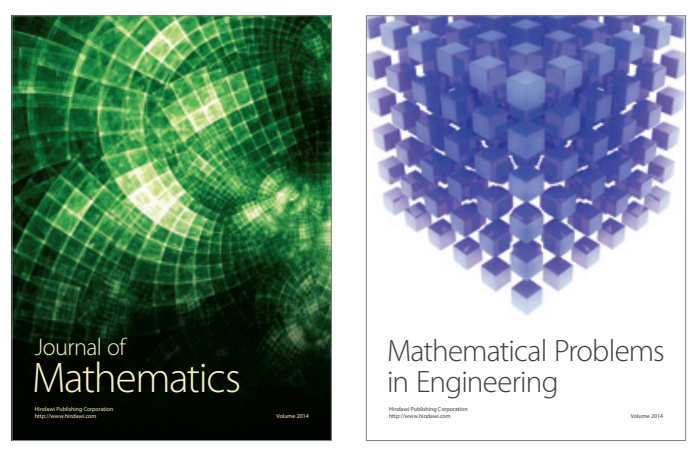

Mathematical Problems in Engineering
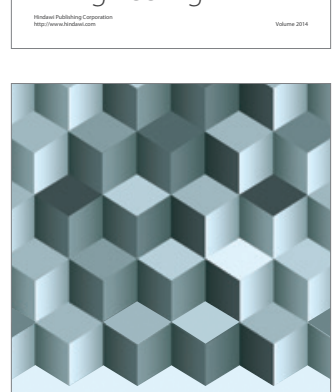

Journal of

Function Spaces
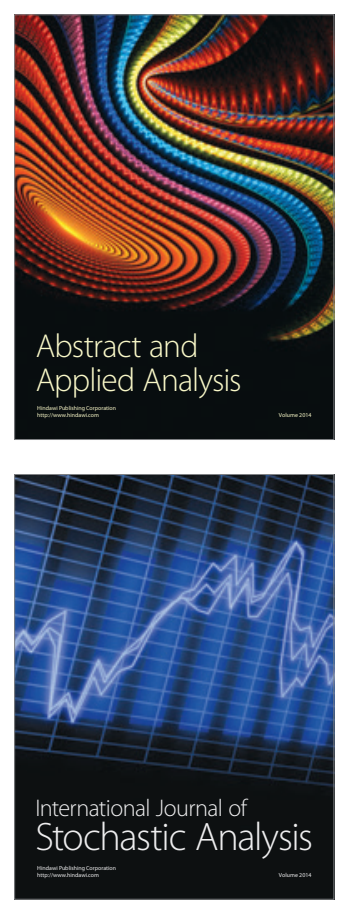

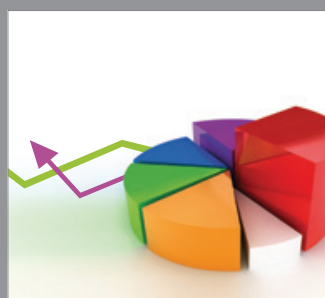

ournal of

Probability and Statistics

Promensencen
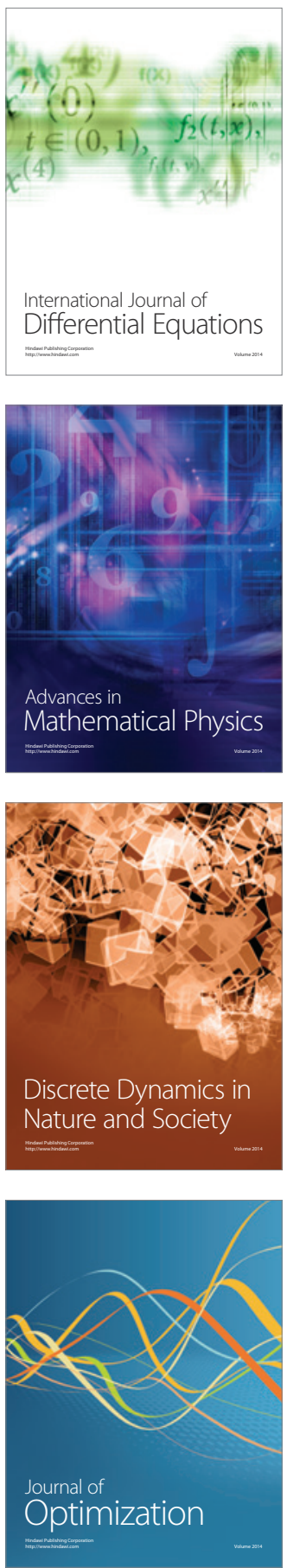